\title{
Practical Applications of Virtual organizations and Agent Technology
}

\author{
Juan M. Corchado ${ }^{1}$, Gabriel Villarrubia ${ }^{1}$, Juan F. De Paz ${ }^{1}$, Sara Rodríguez ${ }^{1}$, Carolina Zato ${ }^{1}$, Fernando \\ de la Prieta ${ }^{1}$ and Javier Bajo ${ }^{2}$ \\ ${ }^{1}$ Departamento Informática y Automática Universidad de Salamanca \\ Plaza de la Merced s/n, 37008, Salamanca, Spain \\ \{corchado;gvg;fcofds;srg;carol_zato;fer\}@usal.es
}

${ }^{2}$ Departamento de Inteligencia Artificial, Universidad Politécnica de Madrid

Campus Montegancedo s/n, 28660, Madrid, Spain

jbajo@fi.upm.es

\begin{abstract}
Computation as interaction paradigm can be considered the most promising technological evolution in the areas of Computer Science and Communication in the last few years. Recent tendencies have conducted to the use of Virtual Organizations (VOs), which can be considered as a set of individuals and institutions that need to coordinate resources and services across institutional boundaries. Multi-agent systems (MAS) technology, which allows forming dynamic agent organizations, is particularly well suited as a support for the development of these open systems. PANGEA is an agent platform to develop open multi-agent systems, specifically those including organizational aspects such as virtual agent organizations. The platform allows the integral management of organizations and offers tools to the end user. Additionally, it includes a communication protocol based on the IRC standard, which facilitates implementation and remains robust even with a large number of connections.
\end{abstract}

Keywords: Multi-agent systems, Virtual Organizations, Dynamic architectures, Adaptive Environments.

\section{Introduction}

Open MAS should allow the participation of heterogeneous agents with different architectures and even different languages [2][1]. The development of open MAS is still a highly research field of the multiagent system paradigm and its development will allow applying the agent technology in new and more complex application domains. However, this makes it impossible to trust agent behaviour unless certain controls based on norms or social rules are imposed. To this end, developers have focused on the organizational aspects of agent societies, using the concepts of organization, norms, roles, etc. to guide the development process of the system.

There are several agent frameworks and platforms [3] [16] [17] that provide a wide range of tools for developing distributed multi-agent systems, however those that allow for the creation of VOs number much fewer, and it is difficult to find one single platform containing all of the requirements for a VO. In conclusion, it could be said that when dealing with all aspects of complex multiagent systems such as VOs, it is also necessary to deal with multiple levels of abstractions and openness, which is not the case for most solutions.

The remainder of the paper is structured as follows: the next section introduces the paradigm of VOs. Section 3 presents an overview of the main characteristics of the platform. Section 4 explains some applications and finally, some conclusions are presented in section 5 . 


\section{Virtual Organizations perspective}

The usefulness of any technology, including multi-agent systems (MAS), can be judged by two criteria: (i) its ability to solve new types of problems and (ii) its ability to improve the efficiency of existing solutions [5]. With this in mind, agents and multi-agent systems provide a natural method of characterizing intelligent systems. Intelligence and interaction are two concepts that are inextricably joined, a fact that is well established in agent technology. When discussing MAS, the idea of a single agent is expanded to include an infrastructure for interaction and communication. Ideally, MAS include the following characteristics [4]: (i) they are typically open with a non-centralized design; (ii) they contain agents that are autonomous, heterogeneous and distributed, each with its own "personality" (cooperative, selfish, honest, etc.). They provide an infrastructure specifically for communication and interaction protocols. Open MAS should allow the participation of heterogeneous agents with different architectures and even different languages [2]. MAS agents based on organizational concepts coordinate and exchange services and information; they are capable of negotiating and coming to an agreement; and they can carry out other more complex social actions. At present, research focusing on the design of MAS from an organizational perspective seems to be gaining most ground. The emergent thought is that modeling the interactions in a MAS cannot be related exclusively to the actual agent and its communication capabilities; instead, organizational engineering is necessary as well. The concepts of rules [7], norms and institutions [8] and social structures [9] are rooted in the idea of needing a higher level of abstraction, independent from the agent, that explicitly defines the organizations in which the agents reside.

Virtual organizations [10] are a means of understanding system models from a sociological perspective. A VO is an open system formed by the grouping and collaboration of heterogeneous entities and there is a separation between form and function that requires defining how a behaviour will take place. The dynamics of open environments is one of the reasons that have encouraged the use of Virtual Organizations of Agents (VOs).

Agent organizations depend on the type of coordination and communication among agents, as well as the type of agents that comprise the group. There are several different organizational approaches [14] [13] [12][2][11][15].

From our perspective, an open platform has been created and allows any type of configuration, adaptation mechanisms, reorganization, search services, etc. All the artifacts that make up a virtual organization can be developed with PANGEA platform.

\section{PANGEA}

PANGEA (Platform for Automatic coNstruction of orGanizations of intElligents Agents) [22] is an agent platform to develop open multi-agent systems, it can manages roles, norms, organizations and suborganizations that facilitate the inclusion of organizational aspects. The services offered by the agents are included completely separate from the agent, facilitating their flexibility and adaption. PANGEA incorporates a CBR-BDI reasoning mechanism available for the agents. The basic agent types defined in PANGEA can be seen in Figure 1, they are:

- OrganizationManager: the agent responsible for the actual management of organizations and suborganizations. It is responsible for verifying the entry and exit of agents, and for assigning roles. To carry out these tasks, it works with the OrganizationAgent, which is a specialized version of this agent.

- InformationAgent: the agent responsible for accessing the database containing all pertinent system information.

- ServiceAgent: the agent responsible for recording and controlling the operation of services offered by the agents. It works as the Directory Facilitator defined in the FIPA standar.

- NormAgent: the agent that ensures compliance with all the refined norms in the organization.

- CommunicationAgent: the agent responsible for controlling communication among agents, and for recording the interaction between agents and organizations.

- Sniffer: manages the message history and filters information by controlling communication initiated by queries.

- DiscoveryAgent: implements an intelligent mechanism to discover services.

- MonitorAgent: interacts with the platform to show the information to the end user. 


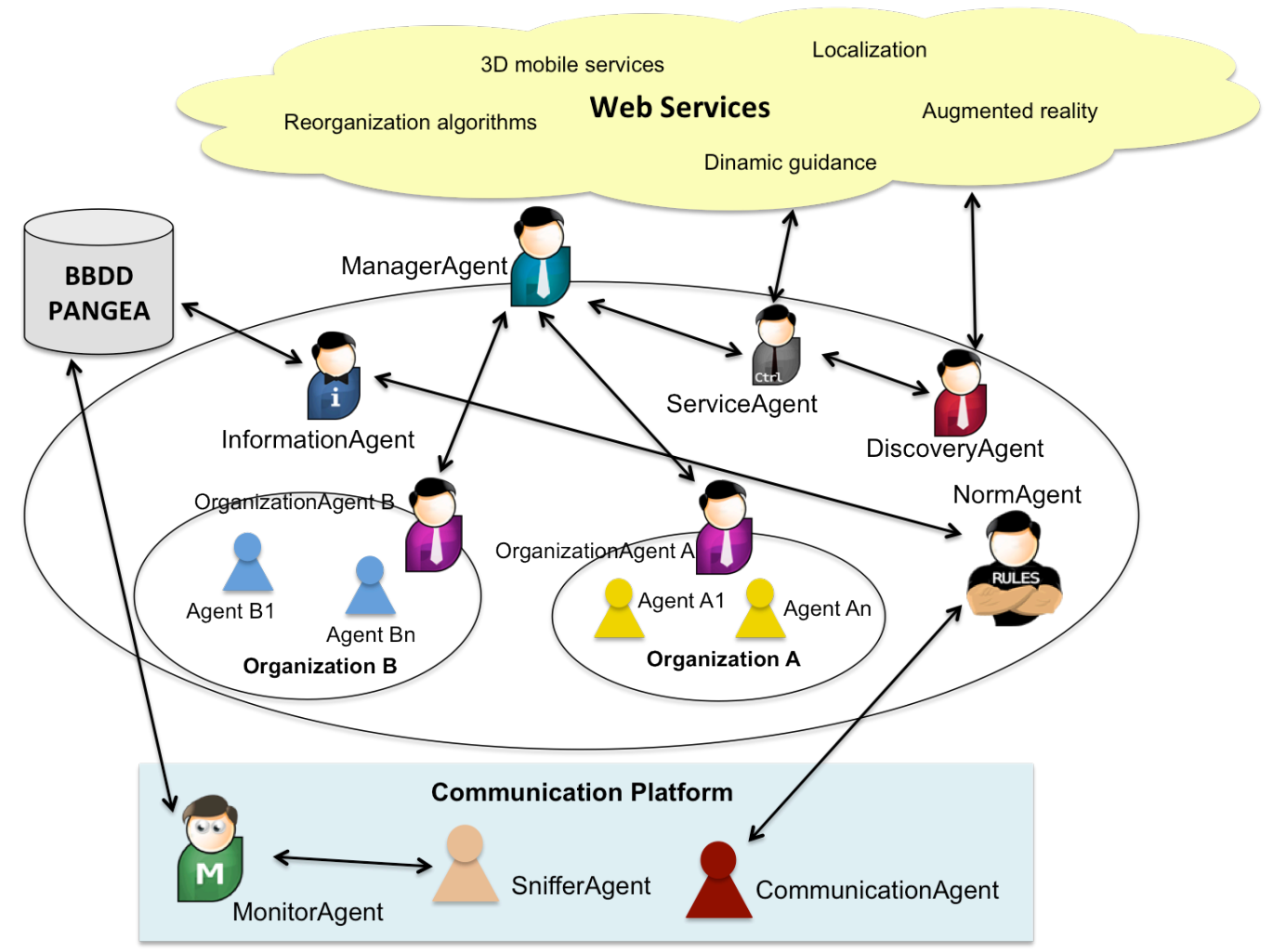

Fig. 1. PANGEA agents

PANGEA is a service-oriented platform that can take maximum advantage of the distribution of resources. To this end, all services are implemented as Web Services. This makes it possible for the platform to include both a service provider agent and a consumer agent, thus emulating a client-server architecture. The provider agent (a general agent that provide a service) knows how to contact the web service, the rest of the agents know how to contact with the provider agent due to their communication with the ServiceAgent, which contains this informacion about services.

Using Web Services also allows the platform to introduce the SOA architecture (Service-Oriented Arquitecture) into MAS systems. SOA is an architectural style for building applications that use services available in a network such as the web. It promotes loose coupling between software components so that they can be reused. Applications in SOA are built based on services. A service is an implementation of a well-defined functionality, and such services can then be consumed by clients in different applications or processes. SOA allows for the reuse of existing services and a level of flexibility that was not possible before in the sense.

One of the most important features that characterize the platform is the use of the IRC protocol for communication among agents. The IRC protocol was used to implement communication. Internet Relay Chat (IRC) is a Real Time Internet Protocol for simultaneous text messaging or conferencing. This protocol is regulated by 5 standards: RFC1459, RFC2810, RFC2811, RFC2812 y RFC2813 [18][19]. The use oh the protocol facilitates the implementation process and provides a flexible and robust communication, already tested by many users. Using IRC involves the ease in implementing communication. The platform's code generating tool makes it possible to easily create an outline of an agent, with the communication code requiring few lines of code. Moreover, it is an open standard protocol that is continuously evolving. There are also IRC clients for all operating systems, including mobile devices. Then, it is important to remark that PANGEA admits mobile agents deployed in Smartphones or tablets and agents in any programming language, it is not necessary to learn a new language in order to use it. 


\section{Applications}

Given the advantages provided by the unique characteristics found in the development of open MAS from an organizational perspective, PANGEA as a VO platform can be use in many different domains.

It has been successfully used to develop many tools and systems. Some examples are: a powerful tool to regulate the elasticity of a Cloud Computing system, different intelligent guidance systems installed in shopping centres or public buildings, management of smart grids, many mobile applications with Augmented Reality for education contexts, a localization and guidance system for planning touristic routes and PANGEA is the main skeleton of all the tools that formed the integral system created in the AZTECA project and the Elderly People Online project.

The AZTECA project [21][23][24] aims to develop new technologies that contribute to the employment of groups of people with visual, hearing or motor disabilities in office environments. Some of these tools are a head mouse to control the mouse with the eyes, a vibrator bracelet to send Morse messages, an avatar for the deaf people, a localization system, etc. The different tools for the disabled people have been modelled with intelligent agents that consume Web services. These agents are implemented and deployed within the PANGEA platform so they form an integral system that can be use regardless of their physical location or implementation.

The Elderly People Online [referencia] intends to develop an intelligent multi-agent system aimed at improving healthcare and assistance to elderly and dependent people in geriatric residences and their homes. The system is implemented using the PANGEA platform and integrates a set of autonomous deliberative agents and planning mechanisms designed to support the caregivers' activities and to guarantee that the patients are given the right care.

\section{Conclusions}

PANGEA facilitates the development of open MAS in an organizational paradigm and has great potential to create open systems highly dynamics. This architecture includes various tools that make it easy for the end user to create, manage and control these systems in aspects such as structural organizations (supporting groups, assuming roles, topology, interactions, and social rules) and the dynamic part of the organization (entry and exit of external agents, creating roles, life cycle of the agents, controlling behaviours, adaption and coordination).

One of the greatest advantages of this system is the communication platform that, by using the IRC standard, offers a robust and widely tested system that can handle a large number of connections, and that additionally facilitates the implementation for other potential extensions. The model used to design PANGEA is very general but without presenting particular restrictions to allow its application to many domains. Its ability to adapt to specific problems does not imply a diminished efficiency in many different applications.

\section{References}

[1] Corchado, E. Pellicer, M. A., Borrajo, M. L. A MLHL Based Method to an Agent-Based Architecture.International Journal of Computer Mathematics. Taylor \& Francis, Volume 86, Issue 10 \& 11, pgs. 1760-1768. 2008 ISI JCR Impact Factor: 0.423

[2] Zambonelli F., N.R. Jennings, and M. Wooldridge. Developing Multiagent Systems: The Gaia Methodology. ACM Transactions on Software Engineering and Methodology, 12:317-370, 2003.

[3] Bravo J., Hervas, R., Chavira, G. and Nava S. Modeling contexts by RFID-sensor fusión. Fourth Annual IEEE International conference on pervasive computing and communications workshops. ISBN: 076952520-25pp-34. Pisa (Italy). 2006

[4] Huhns, M., Stephens, L.: Multiagent Systems and Societies of Agents. In:Weiss, G.(Ed.), Multi-agent Systems: a Modern Approach to Distributed Artificial Intelligence, MIT, 1999.

[5] Jennings, N. \& Wooldridge, M. (eds.), Agent Technology:Foundations, Applications and Markets, Springer, 1998

[6] Zambonelli F., N.R. Jennings, and M. Wooldridge. Developing Multiagent Systems: The Gaia Methodology. ACM Transactions on Software Engineering and Methodology, 12:317-370, 2003.

[7] Zambonelli, F.. Abstractions and Infrastructures for the Design and Development of Mobile Agent Organizations. In: Wooldridge, M., Weiss, G., Ciancarini P. (Eds.): Agent-Oriented Software Engineering II, LNCS 2222, Springer- Verlag, pp. 245 - 262. 2002. 
[8] Esteva M. Electronic Institutions: from specification to development Ph. D. Thesis, Technical University of Catalonia, 2003.

[9] Parunak, H. V. D. and Odell, J. Representing Social Structures in UML. In: Wooldridge, M., Weiss, G., Ciancarini P. (Eds.): Agent-Oriented Software Engineering II, LNCS 2222, Springer. 2002.

[10] Ferber, O. Gutknecht, F. Michel. 2004. From Agents to Organizations: an Organizational View of MultiAgent Systems, in: P. Giorgini, J. Muller, J. Odell (Eds.), Agent-Oriented Software Engineering VI, Vol. LNCS 2935 of Lecture Notes in Computer Science, Springer-Verlag: 214-230

[11] Omicini, A., Ricci, A. and Viroli, M. Coordination artifacts: Environment-based coordination for intelligent agents. Proceedings of 3rd international Joint Conference on Autonomous Agents and Multiagent Systems (AAMAS 2004), 286-293, 2004.

[12] Pavon, J. and Gomez-Sanz, J. J. Agent oriented software engineering with ingenias. Procedings of CEECMAS, volume 2691, pages 394-403. 2003.

[13] Dignum. V. A model for organizational interaction: based on agents, founded in logic, PhD. Thesis, 2004.

[14] Boissier, O. and Gateau, B. Normative multi-agent organizations: Modeling, support and control. Normative Multiagent Systems. 2007.

[15] Hubner, J.F., Sichman, J.S., Boissier, O.: Using the Moise+ for a cooperative framework of mas reorganisation. In: LNAI Proc. of the 17th Brazilian Symposium on Artificial Intelligence (SBIA'04). Volume 3171, 506-515, Springer, 2004.

[16] Stephane Galland. JANUS: Another Yet General-Purpose Multiagent Platform. Seventh AOSE Technical Forum, Paris 2010.

[17] Bordini, R. H., Hübner, J. F., and Vieira, R. 2005. Jason and the Golden Fleece of agent-oriented programming. In Bordini, R. H., Dastani, M., Dix, J., and El Fallah Seghrouchni, A., eds., Multi-Agent Programming: Languages, Platforms and Applications. Springer-Verlag. chapter 1, 3-37

[18] Oikarinen, J. and D. Reed, "Internet Relay Chat Protocol", RFC 1459, May 1993.

[19] Kalt, C., "Internet Relay Chat: Client Protocol", RFC 2810, 2811, 2812, 2813. April 2000.

[20] Chen, M., Kwon, T., Yuan, Y., Choi, Y., Leung, V.C.M. (2007)Mobile agent-based directed diffusion in wireless sensor networks, EURASIP J. Appl. Signal Process, pp.219-219.

[21] AZTECA Project. http://www.tecnologiasaccesibles.com/es/azteca_index.htm

[22] Carolina Zato, Gabriel Villarrubia, Alejandro Sánchez, Ignasi Barri, Edgar Rubión, Alicia Fernández, Carlos Rebate, José A. Cabo, Téresa Álamos, Jesús Sanz, Joaquín Seco, Javier Bajo y Juan M. Corchado. PANGEA - Platform for Automatic coNstruction of orGanizations of intElligent Agents. Distributed 9th International Conference Computing and Artificial Intelligence. 2012. Sigeru Omatu, Juan F. De Paz, Sara Rodríguez,Jose M. Colina, Ana Bernardos y Juan M. Corchado (Eds.)

[23] Alejandro Sánchez, Gabriel Villarrubia, Antonia Macarro, Amparo Jiménez, Carolina Zato, Javier Bajo, Sara Rodríguez, Kasper Hallenborg y Juan M. Corchado. Learning languages using the mobile devices' accelerometer

International Workshop on evidenced-based Technology Enhanced Learning 2012. Pierpaolo Vittorini, Rosella Gennari, Ivana Marenzi, Fernando De la Prieta y Juan M. Corchado (Eds.).

[24] Gabriel Villarrubia, Alejandro Sánchez, Ignasi Barri, Edgar Rubión, Alicia Fernández, Carlos Rebate, José A. Cabo, Téresa Álamos, Jesús Sanz, Joaquín Seco, Carolina Zato, Javier Bajo, Sara Rodríguez and Juan M. Corchado. Proximity detection prototype adapted to a work environment. 3rd International Symposium on Ambient Intelligence (ISAmI 2012). Paulo Novais, Kasper Hallenborg, Dante I. Tapia and Juan M. Corchado (Eds.). 